\title{
Clinical and functional significance of tumor/stromal ATR expression in breast cancer patients
}

\author{
Mysoon M. Al-Ansari ${ }^{1,2}$, Maher Al-Saif ${ }^{3}$, Maria Arafah ${ }^{4}$, Abdelmonneim M. Eldali ${ }^{5}$, Asma Tulbah ${ }^{6}$, Taher Al-Tweigeri ${ }^{7}$,
} Abdelhabib Semlali ${ }^{8}$, Khalid S. Khabar ${ }^{3}$ and Abdelilah Aboussekhra ${ }^{*^{*}}$ (D)

\begin{abstract}
Background: Most breast cancer-associated fibroblasts (CAFs) are active and important cancer-promoting cells, with significant impact on patient prognosis. Therefore, we investigated here the role of the protein kinase ATR in breast stromal fibroblasts in the prognosis of locally advanced breast cancer patients.

Methods: We have used immunohistochemistry to assess the level of ATR in breast cancer tissues and their adjacent normal tissues. Immunoblotting as well as quantitative RT-PCR were utilized to show the role of breast cancer cells and IL-6 as well as AUF-1 in downregulating ATR in breast stromal fibroblasts. Engineered human breast tissue model was also used to show that ATR-deficient breast stromal fibroblasts enhance the growth of breast cancer cells.

Results: We have shown that the protein kinase ATR is downregulated in cancer cells and their neighboring CAFs in breast cancer tissues as compared to their respective adjacent normal tissues. The implication of cancer cells in ATR knockdown in CAFs has been proven in vitro by showing that breast cancer cells downregulate ATR in breast fibroblasts in an IL-6/STAT3-dependent manner and via AUF-1. In another cohort of 103 tumors from locally advanced breast cancer patients, we have shown that absence or reduced ATR expression in tumoral cells and their adjacent stromal fibroblasts is correlated with poor overall survival as well as disease-free survival. Furthermore, ATR expression in CAFs was inversely correlated with tumor recurrence and progression.
\end{abstract}

Conclusion: ATR downregulation in breast CAFs is frequent, procarcinogenic, and correlated with poor patient survival.

Keywords: ATR, AUF-1, Breast cancer, Cancer-associated fibroblasts, Disease-free survival

\section{Background}

While preoperative or neoadjuvant chemotherapy is the mainstay therapeutic strategy for locally advanced breast cancer tumors, it allows disappearance of the tumor (pathological complete response: PCR) in only $20-30 \%$ of breast cancer cases [1]. This prompted several

\footnotetext{
* Correspondence: aboussekhra@kfshrc.edu.sa

'Department of Molecular Oncology, King Faisal Specialist Hospital and Research Center, MBC\#03, Riyadh 11211, Saudi Arabia

Full list of author information is available at the end of the article
}

researchers to search for genes and gene signatures that can predict the response to neoadjuvant therapy of breast cancer. These tumors are heterogeneous and composed of different types of cells, including fibroblasts. Cancer-associated fibroblasts (CAFs), which are the most active stromal cells, play major roles in the development and spread of breast cancer. Although these cells are not cancerous, they exhibit several features of cancer cells, such as high expression level of oncogenes, reduced level of tumor suppressor genes, and high

C C The Author(s). 2020 Open Access This article is licensed under a Creative Commons Attribution 4.0 International License, which permits use, sharing, adaptation, distribution and reproduction in any medium or format, as long as you give appropriate credit to the original author(s) and the source, provide a link to the Creative Commons licence, and indicate if changes were made. The images or other third party material in this article are included in the article's Creative Commons licence, unless indicated otherwise in a credit line to the material. If material is not included in the article's Creative Commons licence and your intended use is not permitted by statutory regulation or exceeds the permitted use, you will need to obtain permission directly from the copyright holder. To view a copy of this licence, visit http://creativecommons.org/licenses/by/4.0/ The Creative Commons Public Domain Dedication waiver (http://creativecommons.org/publicdomain/zero/1.0/) applies to the data made available in this article, unless otherwise stated in a credit line to the data. 
proliferative and invasive capacities [2, 3]. Furthermore, several lines of evidence showed the presence of clear correlation between CAF-related gene signature and clinical outcome of breast cancer patients. These findings suggested an important role of stromal biology in tumor behavior and the consequent patient response to therapy [4].

DNA damage and replication stress are major sources of genomic instability and carcinogenesis [5]. The ataxia telangiectasia and Rad3-related protein (ATR) is a key protein kinase, which plays major roles in the cellular responses to these genotoxic stresses [6]. Indeed, ATR induces various genes and processes that allow cells to cope specifically with the different stresses and insults, in a timely manner. ATR induces cell cycle delay and promotes various DNA repair processes [7]. Furthermore, we have recently shown that ATR level is lower in cancer-associated fibroblasts as compared to their corresponding tumor counterpart fibroblasts (TCFs), and ATR inhibits the procarcinogenic effects of CAFs in a p53-dependent manner [8].

In the present study, we addressed the causes and consequences of ATR downregulation in breast stromal fibroblasts and the correlation between the ATR level and the clinical outcome of breast cancer patients. We have shown AUF1-dependent downregulation of ATR in CAF cells, and poor prognosis of patients bearing tumors expressing low level of ATR in both cancer cells as well as cancer-associated fibroblasts.

\section{Materials and methods}

\section{Cells and cell culture}

Breast fibroblast cells were obtained and used as previously described [9]. MDA-MB-231 and MCF-10A cells were purchased in 2011 from ATCC and were authenticated using short tandem repeat profiling by ATCC, propagated, expanded, and frozen immediately into numerous aliquots after arrival. The revived cells were utilized within 10 to 12 passages and not exceeding a period of 3 months and were cultured following the instructions of the company. Cells were regularly screened for mycoplasma contamination using MycoAlert Mycoplasma Detection Kits (Lonza). All supplements were obtained from Sigma (Saint Louis, MO, USA) except for antibiotic and antimycotic solutions, which were obtained from Gibco (Grand Island, NY, USA). Cells were maintained at $37^{\circ} \mathrm{C}$ in a humidified incubator with $5 \% \mathrm{CO}_{2}$.

Human IL-6 recombinant protein (hBA-184) (Santa Cruz, CA).

\section{Cellular lysate preparation and immunoblotting}

This has been performed as previously described [10]. Antibodies directed against AUF1 (ab50692) and ATR (ab54793) were purchased from Abcam (Cambridge, MA), STAT3 and pSTAT3-Tyr705 (D3A7) from Cell Signaling (Danvers, MA), and glyceraldehydes-3phosphate dehydrogenase (GAPDH, FL-335) from Santa Cruz (Santa Cruz, CA).

\section{RNA purification and qRT-PCR}

Total RNA was purified using the TRI reagent (Sigma) according to the manufacturer's instructions and was treated with RNase-free DNase before cDNA synthesis using the RT-PCR Kit (Clontech, USA). cDNA was amplified using the Platinum ${ }^{\circ}$ Taq DNA Polymerase (Invitrogen). The $\mathrm{RT}^{2}$ Real-Time ${ }^{\mathrm{Tm}}$ SYBR Green $\mathrm{qPCR}$ mastermix (Roche, Germany) was used, and the amplifications were performed utilizing the light cycler 480 (Roche, Germany). The melting-curve data were collected to check PCR specificity, and the amount of PCR products was measured by threshold cycle $(\mathrm{Ct})$ values and the relative ratio of specific genes to $G A P D H$ for each sample was then calculated. The respective primers are:

GAPDH: 5'-GAGTCCACTGGCGTCTTC-3' and 5'GGGGTGCTAAGCAGTTGGT-3'.

ATR: 5' -GTCATATACACTCCCTTTTCTTTA-3' and 5'-GTCATATACACTCCCTTTTCTTTA-3'.

CDKN1A: 5' -CAGAGGAGGCGCCAAGACAG-3' and 5'-CCTGACGGCGGAAAACGC-3'.

CDKN2A: 5' -CAACGCACCGAATAGTTACG-3' and 5'-CAGCTCCTCAGCCAGGTC-3'.

\section{siRNA transfection}

IL-6-siRNA, STAT3-siRNA, and control-siRNA were obtained from QIAGEN, USA. AUF1-siRNA (pSILENCER-AUF15), which targets all four AUF1 isoforms [11], was a generous gift from Dr. Gorospe. The transfections were carried out using the RNAi Fect reagent (QIAGEN) as recommended by the manufacturer.

\section{ATR-shRNA transfection}

ATR-shRNA (KHD1318) expressed in sure silencing shRNA plasmid and the corresponding control plasmid were obtained from GenScript Corporation and were used to carry out transfection using human dermal fibroblast nucleofector 2000 transfection kit (Invitrogen, USA) following the protocol recommended by the manufacturer.

\section{Analysis of mRNA stability}

Cells were challenged with actinomycin $\mathrm{D}(5 \mu \mathrm{g} / \mathrm{ml})$ for various periods of time $(0-6 \mathrm{~h})$, and then total RNA was purified and assessed using qRT-PCR. One-phase exponential decay curve analysis (Sigma Plot) was used to assess the mRNA decay kinetics, considering the values at 
time 0 as $100 \%$. The time corresponding to $50 \%$ remaining mRNA was considered as mRNA half-life.

\section{Conditioned media}

Cells were cultured in medium without serum for $24 \mathrm{~h}$, and then media were collected and briefly centrifuged. The resulting supernatants were used either immediately or were frozen at $-80^{\circ} \mathrm{C}$ until needed.

\section{Cloning of ARE reporter constructs}

The ATR3 3'UTRs were amplified from human fibroblast cDNA by RT-PCR using forward and reverse primers. The forward primer sequence is GGACTCCATATATG TGAAAT and the reverse primer is GTATTAAGAA AGCAGTTT. The primers were designed to contain (G/ GATCC) BamHI and (T/CTAGA) XbaI overhangs. The PCR products were cloned in BamH1 and $\mathrm{XBaI}$ sites in the 3'UTR of a post-transcriptional reporter comprising RPS30 ribosomal promoter and nanoluciferase as previously described $[12,13]$.

\section{Transfection and reporter activity measurements}

Cells were plated at $4.10^{5}$ cells $/ \mathrm{ml}$ per well in a 96-well plate and co-transfected with nanoluciferase posttranscriptional reporter containing the ATR 3'UTR sequences and control non-ARE 3'UTRs that was fused with firefly luciferase. The cells were transfected using Lipofectamine 2000 (Invitrogen) according to the manufacturer's instructions. Transfections were performed in several replicates. After $16 \mathrm{~h}$, cells were lysed and assayed for nanoluciferase activity using the dual NanoGlo dual-luciferase assay kit (Promega) according to the manufacturer's instructions and measured on a luciferase luminometer. Data were presented as mean \pm SEM of normalized Nano-luciferase intensity/firefly intensity.

\section{Engineered human breast tissue (EHBT)}

This has been performed as previously described [14, 15]. Briefly, the lamina propria was produced by mixing rat tail collagen type I ( $3 \mathrm{mg} / \mathrm{mL})$ (Sigma, St. Louis, MO) with breast fibroblasts in fetal calf serum-supplemented culture medium for 4 days. This connective tissue was seeded with MDA-MB-231 to obtain the EHBT and was grown under submerged conditions until the total surface of the connective tissue is covered with epithelial cells. To produce a stratified epithelium, the EHBT was raised to an air-liquid interface for five more days to enable the epithelium to organize into its different strata including the basal layer.

\section{Immunohistochemistry staining on EHBT}

Engineered human breast tissue was cut into 3- $\mu \mathrm{m}$-thick sections, was mounted on saline-coated slides, and was incubated for 15 to $20 \mathrm{~min}$ in a hot air oven at $60^{\circ} \mathrm{C}$.
Slides were then washed and were incubated for $32 \mathrm{~min}$ at $37^{\circ} \mathrm{C}$ with anti-Cyclin D1 and anti-Ki67 (diluted 1: 200) and followed by an incubation with the secondary antibody ultraview universal HRP multimer as previously described [16]. The immunolocalized Ki67 and Cyclin D1 proteins were analyzed by an Olympus BX51 light microscope and DP72 Olympus digital camera (magnification $\times 200$ and $\times 400)$ (Olympus America Inc., Center Valley, PA, USA).

\section{Patients and archived clinical materials}

Formalin-fixed paraffin-embedded tissues were obtained from the Pathology Department at KFSH\&RC with institutional review board approval (RAC\#2160005). The study cohort consisted of 103 locally advanced breast cancer patients who were diagnosed between 2006 and 2013, with a median follow-up time of 52.6 months. Written informed consent was not required and a waiver was granted since samples were anonymized to the research team.

\section{Immunohistochemistry staining on FFPE tissues}

Immunohistochemistry for ATR was done on formalinfixed, paraffin-embedded tissue using anti-ATR from Abcam company (ab54793) overnight at a dilution of 1 : 500, and they were stained using automated staining platform (Ventana). Envision + polymer (ready to use; Dako) was used as a secondary antibody. Color was developed with 3,3'-diaminobenzidine (DAB), and instant hematoxylin (Shandon) was used for counterstaining. The ATR level was evaluated and verified by two qualified pathologists, who scored both the proportion of positive cells as well as the intensity of ATR expression in both cancer cells and their stromal fibroblasts.

\section{Statistical analysis}

Statistical analysis was performed by the software package SAS version 9.4 (SAS Institute Inc., Cary, NC, USA). Continuous variables were compared by Student's $t$ test, and $P$ values of 0.05 and less were considered as statistically significant. The Kaplan-Meyer method was used in survival tables and curves, and the different subgroups were compared by the log-rank test.

\section{Results}

ATR is downregulated in active breast cancer-associated fibroblasts

We started the present study by assessing the ATR expression level in 10 paraffin-embedded sections from breast cancer tissues, including both tumor as well as their adjacent histologically normal tissues, by immunostaining using anti-ATR antibody. Figure la shows a sharp decrease in the ATR protein level in both fibroblasts and tumor cells present in tumors as compared to 


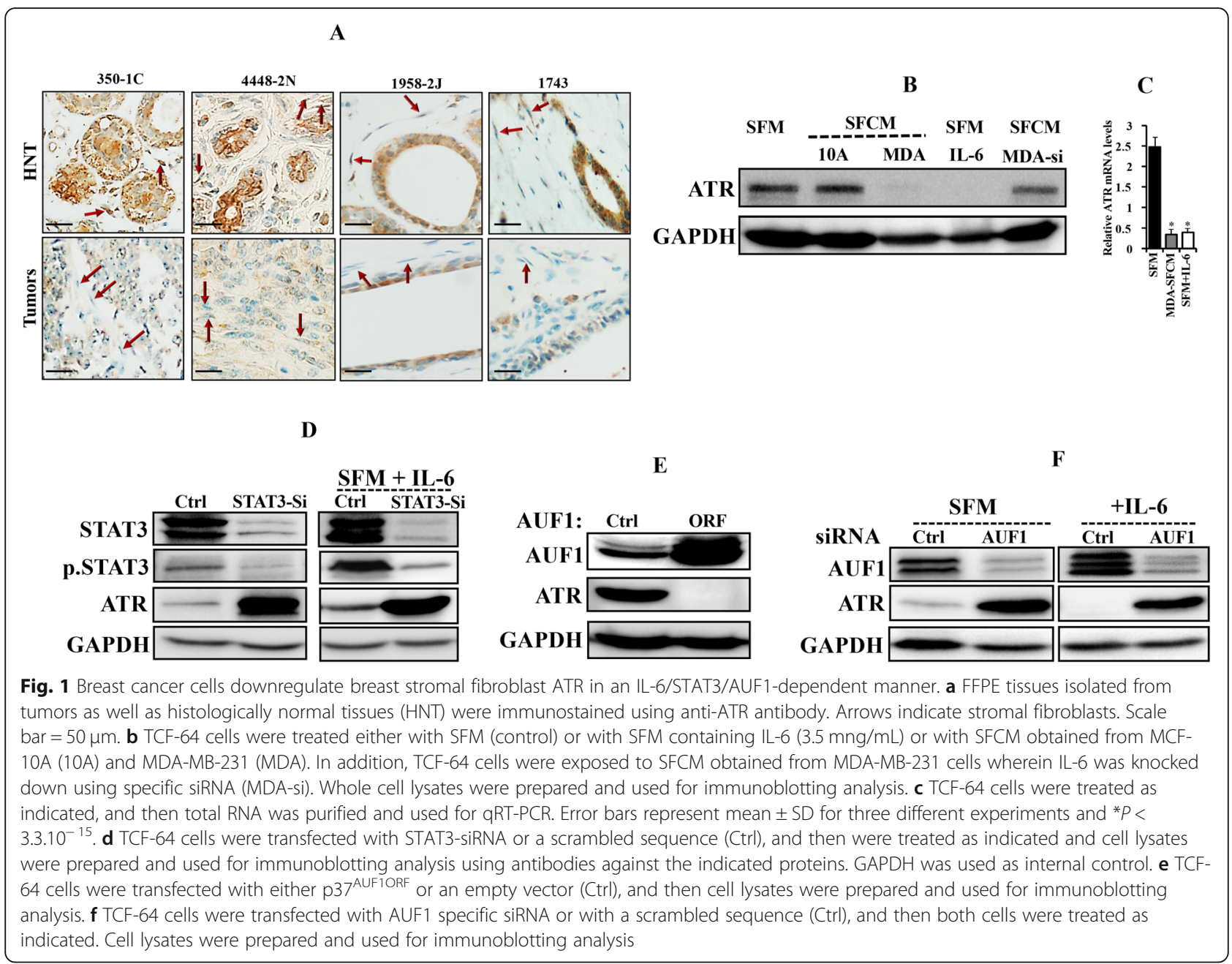

their counterparts present in normal adjacent tissues. This differential expression was obtained in 9 out of 10 pairs, while in the 10th pair, the ATR level was similarly low in both tumor and normal tissues. In 7 out of 10 cases, the level of ATR in stromal fibroblasts was matching the level of ATR in the corresponding cancer cells. This indicates that ATR is downregulated in cancer cells as well as in their neighboring CAFs in breast cancer tissues as compared to their respective adjacent normal tissues.

\section{Breast cancer cells downregulate breast stromal fibroblast} ATR in an IL-6/STAT3/AUF1-dependent manner

We have recently shown that breast cancer cells activate breast stromal fibroblasts (BSFs) in an IL-6-dependent manner [17]. Therefore, we sought to investigate the possible implication of breast cancer cells in ATR downregulation in BSFs through IL-6 signaling. To this end, TCF-64 cells (derived from histologically normal breast cancer tissue) were cultured with serum-free medium (SFM) used as negative control, and serum-free conditioned media (SFCM) derived from both the noncarcinogenic epithelial cells MCF-10A (used as control) as well as the highly invasive breast cancer cells MDAMB-231. Cells were harvested after $24 \mathrm{~h}$, and whole cell lysates were prepared and used for immunoblotting analysis utilizing specific antibodies, and GAPDH was used as internal control. Figure 1b shows that while SFCM from MCF-10A cells had only marginal effect on the ATR level, SFCM from MDA-MB-231 cells abolished ATR expression as compared to the basal level (SFM). Interestingly, adding IL-6 $(3.5 \mathrm{ng} / \mathrm{mL})$ to SFM had an effect similar to that of SFCM from MDA-MB-231 cells (Fig. 1b). Similarly, SFCM from MDA-MB-231 cells and recombinant IL-6 potently reduced the $A T R$ mRNA level (Fig. 1c). On the other hand, IL-6 knockdown by specific siRNA in MDA-MB-231 cells suppressed the paracrine suppressive effect of these cells on the expression of ATR in BSFs (Fig. 1b). This indicates that breast cancer cells downregulate ATR in BSFs in an IL-6-dependent manner. To show the possible implication of STAT3 in this process, we downregulated STAT3 by specific 
siRNA in TCF-64 cells, which strongly upregulated ATR and abolished IL-6-related ATR downregulation (Fig. 1d). Since IL-6-dependent ATR downregulation occurred at the mRNA level and AUF1 is a major posttranscriptional regulator and downstream effector of STAT3 in breast fibroblasts [17], we tested the effect of ectopic expression of $\mathrm{p} 37^{\mathrm{AUF} 1}$ in TCF-64 cells on the expression of ATR. An empty vector was used as negative control. Figure 1e shows AUF1-dependent ATR downregulation. To further elucidate the role of AUF1 in ATR expression in response to IL-6 in BSFs, AUF1 was knocked down by specific siRNA (N64F1si) (a scrambled sequence was used as control) (N64Ctrl), and then cells were exposed either to SFM or SFM containing IL-6. Figure 1f shows that AUF1 downregulation strongly increased the ATR level and also inhibited the IL-6-related ATR downregulation. This shows that IL-6-related ATR inhibition in BSFs is AUF1-dependent and that AUF1 represses the expression of ATR.

\section{AUF1 destabilizes the ATR mRNA through binding its 3' UTR}

To delineate the molecular link between AUF1 and ATR, we tested the possible implication of AUF1 in the turnover of the ATR transcript. Therefore, control and ATR-deficient cells were treated with actinomycin D for various periods of time, and then the proportion of the remaining ATR mRNA was assessed by quantitative RTPCR (qRT-PCR). Figure 2a shows that the ATR mRNA half-life is $3 \mathrm{~h} 30 \mathrm{~min}$ in control cells and that AUF1 knockdown strongly stabilized the $A T R$ transcript. This indicates that AUF1 plays a major role in the ATR mRNA turnover. To confirm this, we first investigated the possible binding of AUF1 to the ATR mRNA. To this end, whole cell lysates were prepared from N64F1si and N64F1Ctrl cells, and then AUF1-mRNA ribonucleoprotein complexes were obtained by IP using anti-AUF1 antibody (IgG was used for control cells) and were used for qRT-PCR. The immunoprecipitation with anti-AUF1

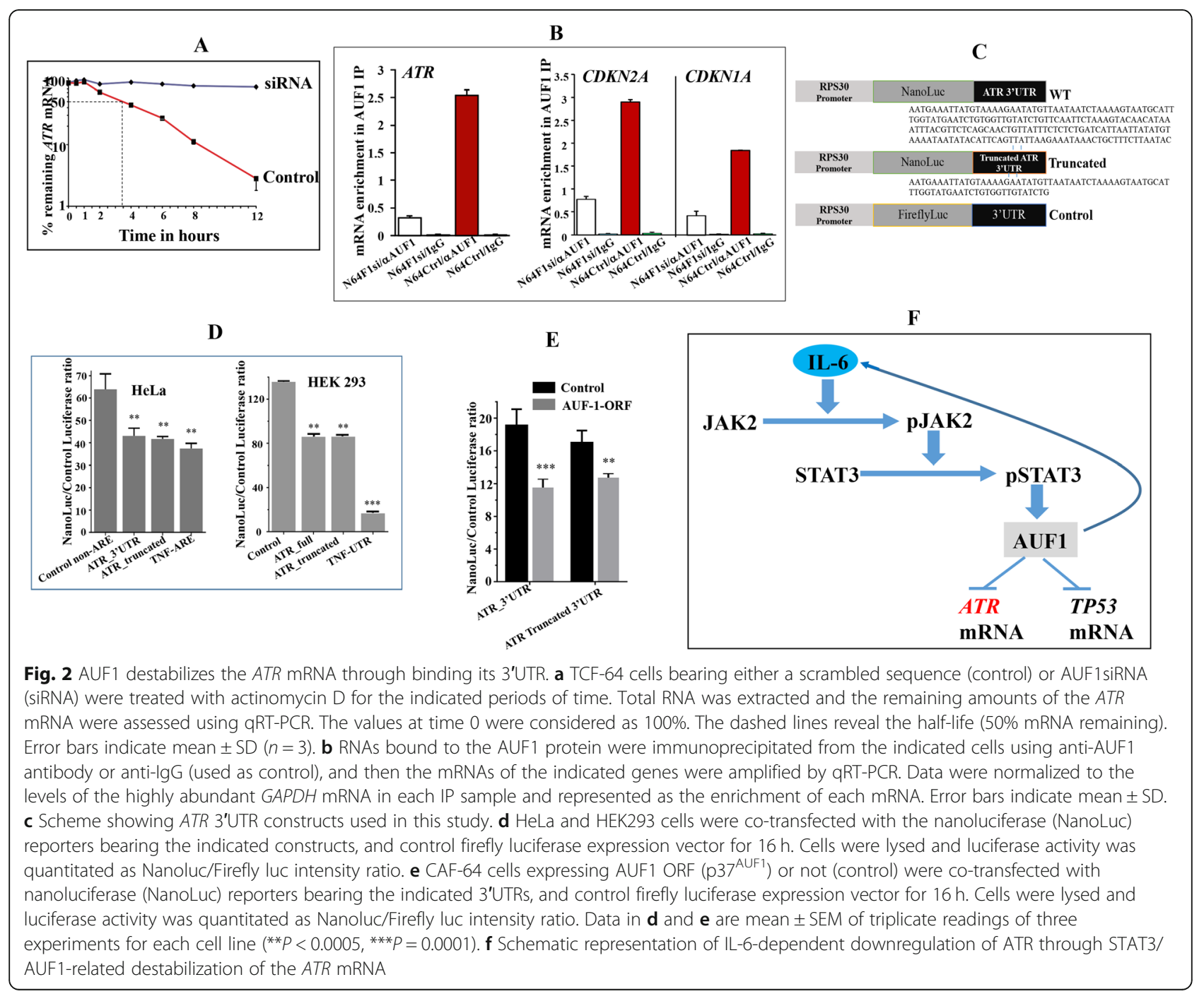


antibody yielded higher amplification of the $A T R$, CDKN1A, and CDKN2A mRNAs in control cells as compared to AUF1-deficient cells (N64F1si) (Fig. 2b). However, only marginal amplifications were obtained following immunoprecipitation with IgG (Fig. 2b). This shows that, like for the CDKN2A and CDKN2B mRNAs, AUF1 binds the ATR mRNA as well. To confirm the AUF1-related destabilization of the ATR mRNA and the implication of its 3'UTR in this interaction, the fulllength ATR 3'UTR and artificially truncated form, along with control non-ARE 3'UTR, were subcloned into a post-transcriptional reporter [12, 13] (Fig. 2c). We found that full ATR 3'UTR caused significant reduction of the post-transcription reporter in both HEK293 and HeLa cell lines (Fig. 2d). Likewise, a truncated form (73-bases) of the ATR 3'UTR caused a similar reduction in both cell lines (Fig. 2d). As positive control, the strong TNF 3'UTR was used, and a highly significant effect was obtained (Fig. 2d). The effect of AUF1 on the full ATR 3' UTR and the truncated 3'UTR was examined in TCF-64 fibroblasts stably expressing $\mathrm{p} 37^{\mathrm{AUF1ORF}}$ or an empty vector used as control. There was statistically significant reduction (nearly 40\%) of the post-transcription reporter with the full-length ATR and to a lesser degree with the truncated form (25\% reduction) (Fig. 2e). Together, these results show that AUF1 binds the ATR 3'UTR and enhances the turnover of the corresponding message. This has been summarized in Fig. $2 f$.

\section{ATR-deficient breast stromal fibroblasts enhance the growth of breast cancer cells in engineered human breast tissue}

After showing the effects of ATR deficiency in 2D cultured cells, we decided to move our study closer to the in vivo setting by using an engineered human breast tissue (EHBT) model, as previously described by Semlali et al. $[14,15]$. In this model, the connective tissue was formed with the TCF-64 cells expressing either specific ATR-shRNA (N64-sh, ATR-deficient) or a control vector (N64C) as previously shown [8], while the epithelium was generated with the highly aggressive and proliferative MDA-MB-231 cells. Figure 3a shows the generated EHBT structures, with striking differences in the organization and the width of the epithelium. Indeed, while the epithelium from the control tissue revealed a disorganized structure with a small epithelial layer, N64sh cells generated a well-organized structure, with larger stratified multilayered epithelial tissue (Fig. 3a). This indicates more proliferative and aggressive breast cancer cells while adjacent to ATR-deficient stromal fibroblasts. This pro-proliferative effect of ATR-deficient cells was confirmed by immunostaining showing an increase in the staining of Ki-67 and cyclin D1 in the epithelium adjacent to N64-sh cells as compared to control (Fig. 3b).

\section{Correlation of ATR expression in cancer as well as stromal fibroblasts with clinicopathological parameters}

Next, we sought to investigate the predictive value of ATR expression levels in cancer cells as well as in stromal fibroblasts as a candidate biomarker for clinical outcome of patients with LABC. The clinicopathological features of the enrolled patients $(n=103)$ are listed in Additional file 1. ER+/Her2+ patients (26) represented 25\%, ER+/Her2- (32) represented 31\%, and ER-/Her+ (24) represented 23\%, while ER-/Her2- (21) represented $20 \%$. Remarkably, $70 \%$ of the patients were less than 50 years old, and the same proportion had high tumor stage, while $52 \%$ of the tumors were of grade 3 . Thirty-

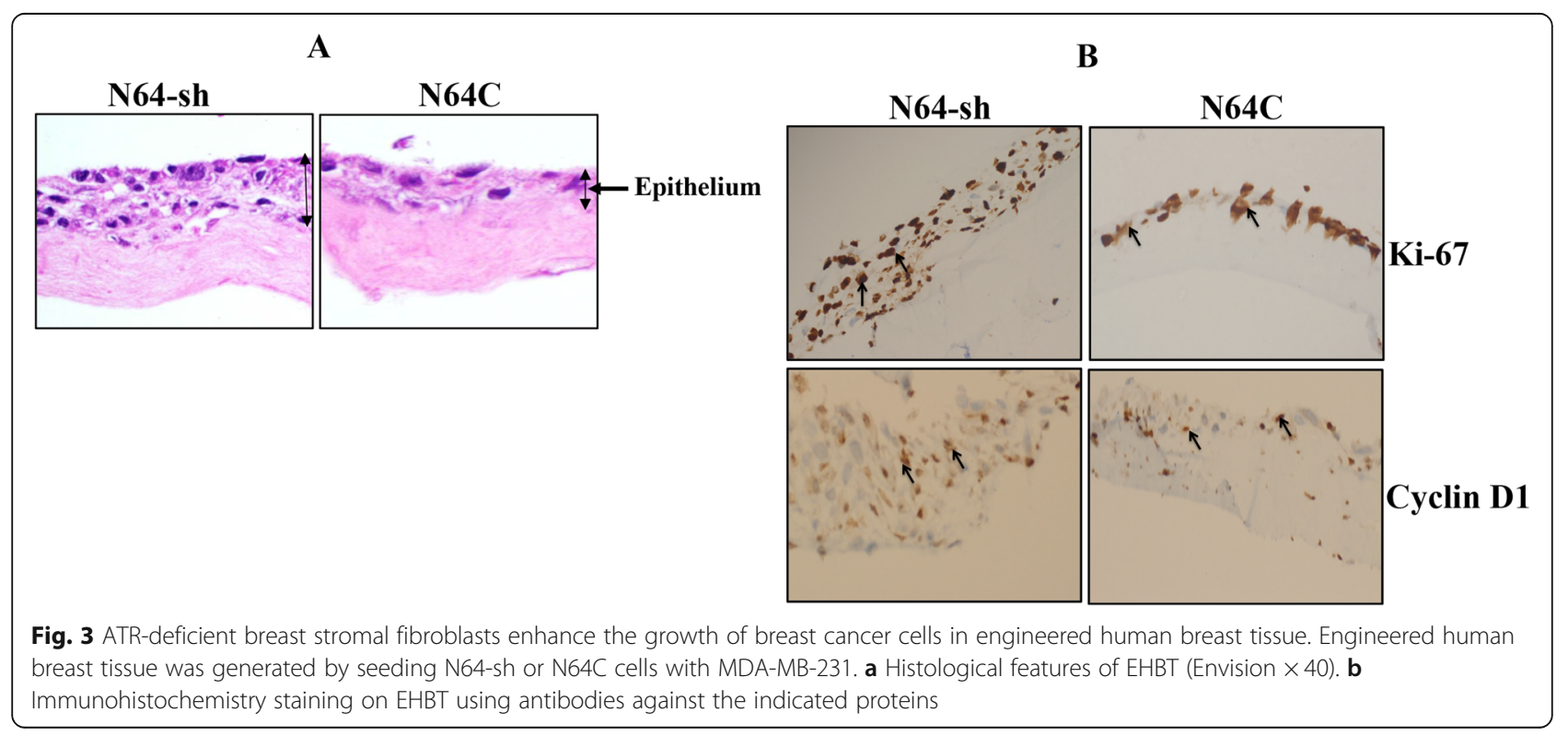


nine patients developed recurrence and 19 died (Additional file 1). Notably, $60 \%$ of patients had tumor sizes more than $5 \mathrm{~cm}$ (Additional file 1).

A total of 103 breast pretreatment tumor tissues were assessed for ATR expression in both cancer as well as stromal cells. The reading of slides was performed and verified by 2 independent qualified pathologists. Figure 4a shows ATR immunostaining in breast cancer tissues in both epithelium and stroma. The ATR immunostaining in both types of cells was classified into three subgroups: low (0-10\% ATR positive cells), intermediate $(11-50 \%$ ATR positive cells), and high (51-100\% ATR positive cells). Additional file 2 shows that the ATR expression level was low or completely lost in 46/103 fibroblast and $38 / 103$ epithelial cells, intermediate in 30/103 fibroblast and 19/103 epithelial cells, and high in 27/103 fibroblast and 46/103 epithelial cells. The ATR expression in the different subtypes indicated association with ER/Her2. Indeed, significant correlation ( $p$ value $=0.0212$ ) was observed between low ATR level in fibroblasts and lack of ER or Her2 (Additional file 2). However, no correlation was observed between ATR level in cancer cells and ER/ Her2 expression levels (Additional file 2). Table 1 shows that the expression level of ATR in CAFs did not correlate with tumor stage and the Ki-67 index. However, ATR expression in CAFs was significantly associated with tumor grade and progression $(P=0.0473$ and $P=$ 0.0003 , respectively) as well as patient survival $(P<$ 0.0001 ), while it was inversely correlated with tumor recurrence $(P=0.0017)$ (Table 1). Patient survival was also highly correlated with the level of ATR in breast cancer cells $(P=0.0006)$ (Table 1$)$.

\section{ATR expression in both breast cancer cells and their stromal fibroblasts predicts survival}

Kaplan-Meier plots shown in Fig. 3b, c indicate significant association between ATR expression levels in both tumoral and stromal cells and patient's overall survival (OS) as well as disease-free survival (DFS). Indeed, patients with low ATR expression in stromal fibroblasts had significantly poorer DFS as well as OS rates (Fig. 4b). In contrast, patients with moderate or high ATR expression levels showed better OS and DFS ( $P=0.0001$ and $P=0.0004$, respectively) (Fig. $4 \mathrm{~b}$ ). Interestingly, patients with moderate levels of stromal ATR showed the best OS and DFS (Fig. 4b). Similarly, the ATR levels in cancer cells were correlated with patient survival (Fig. 4c). In these cases, patients with moderate and high ATR levels showed similar higher OS and DFS than patients with low ATR expression $(P=0.0006$ and $P=0.0392$, respectively $)$ (Fig. 4c). Univariate Cox regression analysis also showed an increased risk for patients with low ATR levels in both stromal fibroblasts (hazard ratio $>1$; $P=0.0001$ ) and cancer cells (hazard ratio $>1 ; P=$ 0.0012) (Table 2). In order to establish whether the prognostic power of the ATR expression level is independent of other well-known breast cancer risk factors, multivariate Cox regression analysis was conducted. Table 3 indicates that the ATR expression level in stromal fibroblasts is a significant independent predictor of OS and DFS $(P=0.0019, P=0.0285$, respectively) (Table 3 ). Similarly, ATR expression in cancer cells was an independent prognostic factor for OS, but not for DFS (Table 3).

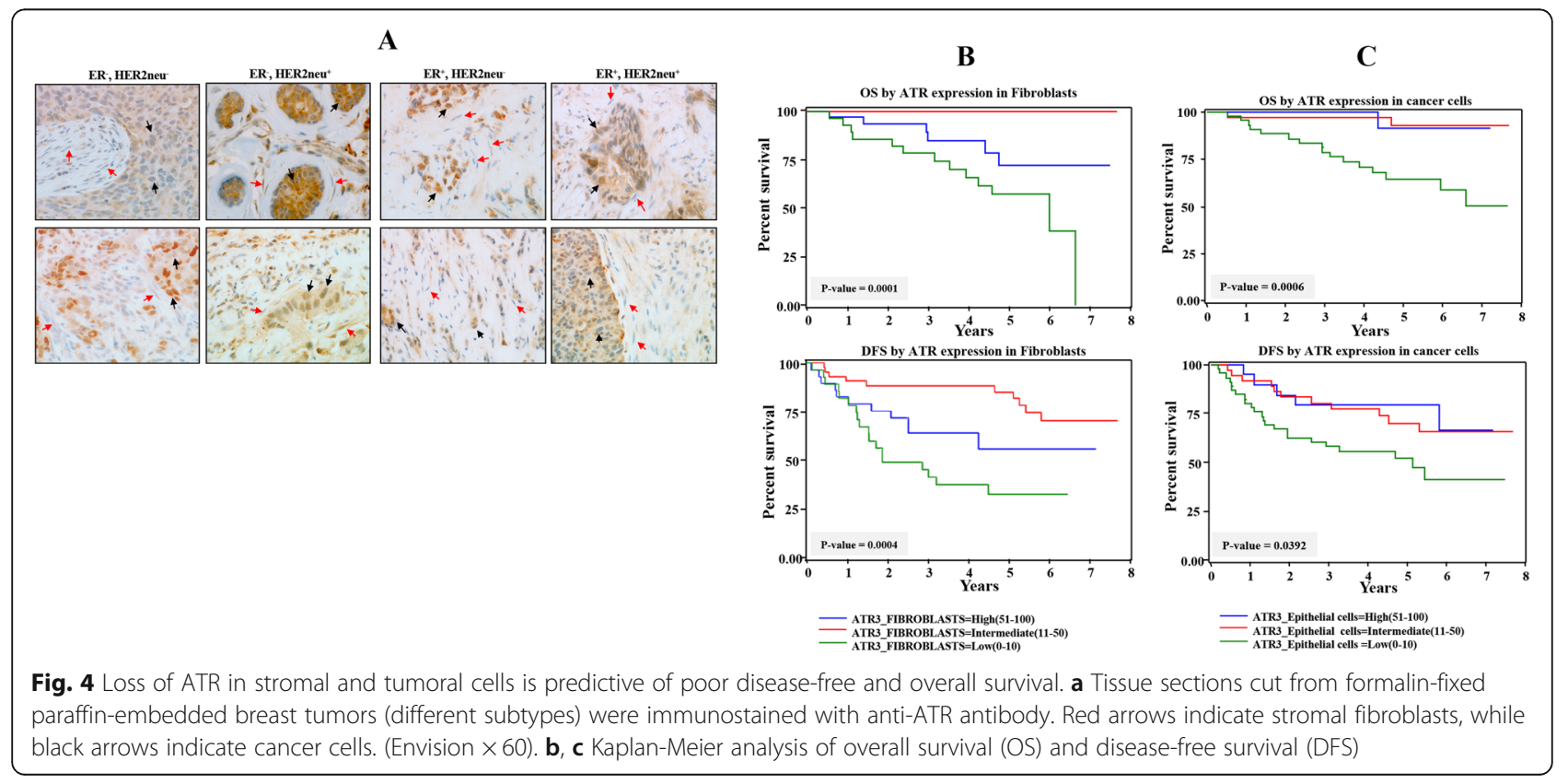


Table 1 Correlations between ATR expression and clinicopathological characteristics in breast cancer patients

\begin{tabular}{|c|c|c|c|c|c|}
\hline \multirow[t]{2}{*}{ Parameter } & \multirow[t]{2}{*}{ Total $(n=103)(\%)$} & \multicolumn{3}{|c|}{ ATR in stromal fibroblasts } & \multirow[t]{2}{*}{$P$ value } \\
\hline & & High & Intermediate & Low & \\
\hline \multicolumn{6}{|l|}{ Stage } \\
\hline $\mathrm{T} 2$ & $30(29.13)$ & $6(5.83)$ & $17(16.50)$ & $7(6.80)$ & \\
\hline T3 & $29(28.16)$ & $10(9.71)$ & $13(12.62)$ & $6(5.83)$ & 0.3308 \\
\hline $\mathrm{T} 4$ & $43(41.75)$ & $14(13.59)$ & $14(13.59)$ & $15(14.56)$ & \\
\hline Tx & $1(0.97)$ & $0(0.00)$ & $1(0.97)$ & $0(0.00)$ & \\
\hline \multicolumn{6}{|l|}{$\mathrm{KI}-67$ index } \\
\hline 0 & $85(84.16)$ & $26(24.75)$ & $37(36.63)$ & $23(22.77)$ & \\
\hline$\leq 15$ & $2(1.98)$ & $0(0.00)$ & $2(1.98)$ & $0(0.00)$ & 0.7652 \\
\hline$>15$ & $14(13.86)$ & $5(4.95)$ & $5(4.95)$ & $4(3.96)$ & \\
\hline \multicolumn{6}{|l|}{ HIS subtype } \\
\hline None invasive & $1(0.97)$ & $1(0.97)$ & $0(0.00)$ & $0(0.00)$ & \\
\hline Invasive ductal Ca & $85(82.52)$ & $23(22.33)$ & $40(38.83)$ & $22(21.36)$ & \\
\hline 1,4 & $3(2.91)$ & $1(0.97)$ & $1(0.97)$ & $1(0.97)$ & 0.5887 \\
\hline Invasive ductal Ca with DCIS & $11(10.68)$ & $3(2.91)$ & $4(3.88)$ & $4(3.88)$ & \\
\hline Infiltrating lobular Ca & $1(0.97)$ & $0(0.00)$ & $0(0.00)$ & $1(0.97)$ & \\
\hline 3,4 & $1(0.97)$ & $1(0.97)$ & $0(0.00)$ & $0(0.00)$ & \\
\hline Other & $1(0.97)$ & $1(0.97)$ & $0(0.00)$ & $0(0.00)$ & \\
\hline \multicolumn{6}{|l|}{ Recurrence } \\
\hline No & $63(61.76)$ & 18 (17.65) & $35(34.31)$ & $10(9.80)$ & 0.0017 \\
\hline Yes & 39 (38.24) & 11 (10.78) & $10(9.80)$ & 18 (17.65) & \\
\hline \multicolumn{6}{|l|}{ Grade } \\
\hline G1 & $3(2.91)$ & $1(0.97)$ & $1(0.97)$ & $1(0.97)$ & \\
\hline G2 & $46(44.66)$ & 9 (8.74) & $27(26.21)$ & $10(9.71)$ & 0.0473 \\
\hline G3 & $54(52.43)$ & $20(19.42)$ & $17(16.50)$ & $17(16.50)$ & \\
\hline \multicolumn{6}{|l|}{ Progression } \\
\hline No & 73 (72.28) & $13(12.87)$ & $40(39.60)$ & $20(19.81)$ & 0.0003 \\
\hline Yes & $28(27.72)$ & $14(13.86)$ & $4(3.96)$ & $18(9.9)$ & \\
\hline \multicolumn{6}{|l|}{ Survival status } \\
\hline Alive & $84(81.55)$ & $24(23.30)$ & $45(43.69)$ & $15(14.56)$ & $<0.0001$ \\
\hline Dead & 19 (18.45) & $6(5.83)$ & $0(0.00)$ & $13(12.62)$ & \\
\hline Duration of clinical follow-up (years, mean $\pm S D$ ) & & 4.16500000 & 5.00755556 & 3.87464286 & 0.0390 \\
\hline \multirow[t]{2}{*}{ Parameter } & Total $(n=103)(\%)$ & \multicolumn{3}{|c|}{ ATR in cancer cells } & $P$ value \\
\hline & & High & Intermediate & Low & \\
\hline \multicolumn{6}{|l|}{ Tumor size } \\
\hline$\leq 5$ & $35(40.23)$ & $7(8.05)$ & $15(17.24)$ & $13(14.94)$ & 0.4008 \\
\hline$>5$ & $52(59.77)$ & $8(9.20)$ & $17(19.54)$ & $27(31.03)$ & \\
\hline \multicolumn{6}{|l|}{ Stage } \\
\hline $\mathrm{T} 2$ & $30(29.13)$ & $6(5.83)$ & $12(11.65)$ & $12(11.65)$ & \\
\hline $\mathrm{T} 3$ & $29(28.16)$ & $6(5.83)$ & $10(9.71)$ & $13(12.62)$ & 0.6772 \\
\hline T4 & $43(41.75)$ & $6(5.83)$ & $16(15.53)$ & $21(20.39)$ & \\
\hline Tx & $1(0.97)$ & $1(0.97)$ & $0(0.00)$ & $0(0.00)$ & \\
\hline \multicolumn{6}{|l|}{$\mathrm{KI}-67$ index } \\
\hline 0 & 85 (84.16) & $13(12.87)$ & $32(31.68)$ & $40(39.60)$ & 0.1525 \\
\hline
\end{tabular}


Table 1 Correlations between ATR expression and clinicopathological characteristics in breast cancer patients (Continued)

\begin{tabular}{|c|c|c|c|c|c|}
\hline$\leq 15$ & $2(1.98)$ & $0(0.00)$ & $1(0.99)$ & $1(0.99)$ & \\
\hline$>15$ & $14(13.86)$ & $6(5.94)$ & $3(2.97)$ & $5(4.95)$ & \\
\hline \multicolumn{6}{|l|}{ HIS subtype } \\
\hline None invasive & $1(0.97)$ & $0(0.00)$ & $1(0.97)$ & $0(0.00)$ & \\
\hline Invasive ductal $\mathrm{Ca}$ & $85(82.52)$ & $13(12.62)$ & $33(32.04)$ & $39(37.86)$ & \\
\hline 1,4 & $3(2.91)$ & $0(0.00)$ & $1(0.97)$ & $2(1.94)$ & \multirow[t]{5}{*}{0.1336} \\
\hline Invasive ductal Ca with DCIS & $11(10.68)$ & 4 3.88) & $2(1.94)$ & $5(4.85)$ & \\
\hline Infiltrating lobular $\mathrm{Ca}$ & $1(0.97)$ & $0(0.00)$ & $1(0.97)$ & $0(0.00)$ & \\
\hline 3,4 & $1(0.97)$ & $1(0.97)$ & $0(0.00)$ & $0(0.00)$ & \\
\hline Other & $1(0.97)$ & $1(0.97)$ & $0(0.00)$ & $0(0.00)$ & \\
\hline \multicolumn{6}{|l|}{ Recurrence } \\
\hline No & $63(61.76)$ & $14(13.73)$ & $26(25.49)$ & $23(22.55)$ & \multirow[t]{2}{*}{0.1000} \\
\hline Yes & $39(38.24)$ & $5(4.90)$ & $11(10.78)$ & $23(22.55)$ & \\
\hline \multicolumn{6}{|l|}{ Grade } \\
\hline G1 & $3(2.91)$ & $1(0.97)$ & $1(0.97$ & $1(0.97)$ & \multirow{3}{*}{0.6015} \\
\hline G2 & $46(44.66)$ & $10(9.71)$ & $14(13.59)$ & $22(21.36)$ & \\
\hline G3 & $54(52.43)$ & $8(7.77)$ & $23(22.33)$ & $23(22.33)$ & \\
\hline \multicolumn{6}{|l|}{ Progression } \\
\hline No & $73(72.28)$ & $17(16.83)$ & $29(28.71)$ & $27(26.73)$ & \multirow[t]{2}{*}{0.0570} \\
\hline Yes & $28(27.72)$ & $2(1.98)$ & $9(8.91)$ & $17(16.83)$ & \\
\hline \multicolumn{6}{|l|}{ Survival status } \\
\hline Alive & $84(81.55)$ & $18(17.48)$ & $36(34.95)$ & $30(29.13)$ & \multirow[t]{2}{*}{0.0006} \\
\hline Dead & $19(18.45)$ & $1(0.97)$ & $2(1.94)$ & $16(15.53)$ & \\
\hline Duration of clinical follow-up (years) & & 4.16500000 & 5.00755556 & 3.87464286 & 0.1883 \\
\hline
\end{tabular}

\section{Discussion}

It has become clear that breast stromal fibroblasts play important roles in tumor spreading and relapse as well as the therapeutic outcome of patients. Furthermore, recent findings underscored CAFs as important potential diagnostic and/or prognostic tool in clinical practice $[3,18,19]$. Therefore, it is of utmost importance to further characterize these cells and determine relevant predictive markers. In the present study, we have shown that the cell cycle protein kinase ATR is lowly expressed in CAFs compared to their corresponding cells present in adjacent histologically normal tissues. This corroborates our previous results obtained in cultured CAF/ TCF cells [8]. This differential expression suggests that ATR downregulation in CAFs is due to the presence of these fibroblasts close to cancer cells, which perturbs the microenvironment through paracrine signaling. In fact, we have recently shown that cancer cells activate breast stromal fibroblasts in an IL-6-dependent manner [20]. Therefore, we tested the effect of breast cancer cells and IL-6 on the expression of ATR in BSFs, and have shown that breast cancer cells as well as pure recombinant IL-6 downregulate ATR in BSFs. This effect was mediated through IL-6-dependent activation of STAT3 and its downstream target AUF1. This prompted us to elucidate the link between AUF1 and ATR and have shown that AUF1 destabilizes the ATR mRNA through binding its 3'UTR and accelerates its turnover, which explains ATR downregulation in active BSFs. This has been summarized in Fig. 2f. Similar AUF1-related post-transcriptional regulation has been previously shown for p16 in breast myofibroblasts [21]. AUF1 is also a repressor of p53 [22], which indicates that AUF1 upregulation is a major step toward BSF transactivation. Indeed, we have previously shown that AUF1 is upregulated in 66\% CAFs relative to their adjacent TCF counterparts [21]. This confirms that ATR is another important target of breast cancer cells in BSFs, and that this protein kinase plays key roles in the carcinomastroma cross-talk during breast carcinogenesis. The procarcinogenic effects of ATR-deficient breast 
Table 2 Univariate Cox proportional regression analysis on 5-year overall and disease-free survival of 103 LABC patients

\begin{tabular}{|c|c|c|c|c|c|c|}
\hline \multirow[t]{2}{*}{ Parameter } & \multicolumn{3}{|c|}{ Overall survival } & \multicolumn{3}{|c|}{ Disease-free survival } \\
\hline & Hazard ratio & $95 \% \mathrm{Cl}+$ & $P^{*}$ & Hazard ratio & $95 \% \mathrm{Cl}+$ & $P^{*}$ \\
\hline \multicolumn{7}{|l|}{ ATR (fibroblasts) } \\
\hline High & 1.000 & & & 1.000 & & \\
\hline Low & 7.934 & $2.950-21.340$ & 0.0001 & 0.406 & $0.198-0.833$ & 0.0140 \\
\hline \multicolumn{7}{|l|}{ ATR (epithelial cells) } \\
\hline High & 1.000 & & & 1.000 & & \\
\hline Low & 7.711 & $2.244-26.498$ & 0.0012 & 0.732 & $0.422-1.270$ & 0.2670 \\
\hline \multicolumn{7}{|l|}{ Tumor size } \\
\hline$\leq 5 \mathrm{~cm}$ & 1.000 & & & 1.000 & & \\
\hline$>5 \mathrm{~cm}$ & 1.239 & $0.439-3.500$ & 0.6852 & 0.796 & $0.452-1.404$ & 0.4311 \\
\hline \multicolumn{7}{|l|}{ Age } \\
\hline$\leq 52$ years & 1.000 & & & 1.000 & & \\
\hline$>52$ years & 0.984 & $0.940-1.030$ & 0.4826 & 1.012 & $0.985-1.039$ & 0.3854 \\
\hline \multicolumn{7}{|l|}{ Stage } \\
\hline $\mathrm{T} 2$ & 1.000 & & & 1.000 & & \\
\hline $\mathrm{T} 3, \mathrm{~T} 4, \mathrm{Tx}$ & 1.941 & $0.642-5.866$ & 0.2401 & 0.508 & $0.292-0.883$ & 0.0163 \\
\hline \multicolumn{7}{|l|}{ Grade } \\
\hline$|/| \mid$ & 1.000 & & & 1.000 & & \\
\hline Ш/poorly differentiated & 1.949 & $0.758-5.012$ & 0.1660 & 1.241 & $0.723-2.130$ & 0.4328 \\
\hline
\end{tabular}

stromal fibroblasts were shown in vitro using direct coculturing in engineered human breast tissues composed of breast cancer cells and BSFs. Indeed, ATRdeficient cells enhanced the growth of breast cancer cells, which was confirmed by showing the expression of high levels of Ki-67 and cyclin D1. Similarly, we have recently shown that ATR downregulation activates BSFs and enhance their paracrine procarcinogenic effects both in vitro and ion orthotopic tumor xenografts [8]. These protumorigenic effects of ATR-deficient breast stromal fibroblasts prompted us to evaluate the consequences of ATR downregulation in cancer cells as well as their stromal fibroblasts on the survival of patients with LABC treated with neoadjuvant therapy, which is widely used for the treatment of these high-risk breast cancer patients. Therefore, identification of better markers of response is highly needed for a better stratification of patients. Importantly, we have shown significant association between ATR expression levels in both tumoral and stromal cells and patient's overall survival as well as disease-free survival. In both cell types, absence or reduced ATR expression predicted poor clinical outcome in locally advanced breast cancers. This indicates that low ATR levels in cancer and/or stromal fibroblasts in these tumors can significantly predict high risk of recurrence.
Interestingly, while moderate and high ATR levels in cancer cells were similarly correlated with better outcome, moderate ATR levels in stromal fibroblasts were correlated with better patient outcome than those who expressed high ATR levels (Fig. 4). Furthermore, ATR expression in CAFs was inversely correlated with tumor recurrence, progression, and patient survival, which was also highly correlated with the level of ATR in breast cancer cells.

Univariate Cox regression analysis confirmed that low ATR levels in both stromal fibroblasts and cancer cells were a significant indicator of poor clinical outcome. Moreover, multivariate Cox regression analysis showed that the ATR expression level in stromal fibroblasts is a significant independent predictor of OS and DFS, while ATR level in cancer cells was an independent prognostic factor only for OS, but not for DFS (Table 3). This indicates that the ATR expression level in both cancer as well as stromal fibroblasts could be of great prognostic value for patients with $\mathrm{LABC}$ treated with neoadjuvant therapy.

\section{Conclusions}

The present findings show that ATR is downregulated in cancer cells and their neighboring CAFs in breast cancer tissues as compared to their respective adjacent normal tissues. Furthermore, we present 
Table 3 Multivariate Cox regression analysis on 5-year overall and disease-free survival

\begin{tabular}{|c|c|c|c|c|c|c|}
\hline \multirow[t]{2}{*}{ Parameter } & \multicolumn{3}{|c|}{ Overall survival } & \multicolumn{3}{|c|}{ Disease-free survival } \\
\hline & Hazard ratio & $95 \% \mathrm{Cl}+$ & $P^{*}$ & Hazard ratio & $95 \% \mathrm{Cl} \dagger$ & $P^{*}$ \\
\hline ATR (fibroblasts) & 14.708 & $2.693-80.332$ & 0.0019 & 0.369 & $0.151-0.900$ & 0.0285 \\
\hline Tumor size & 0.665 & $0.084-5.244$ & 0.6985 & 1.018 & $0.276-3.759$ & 0.9790 \\
\hline Age & 0.878 & $0.802-0.962$ & 0.0051 & 1.027 & $0.989-1.067$ & 0.1642 \\
\hline Stage & 95.600 & 5.109-1788.693 & 0.0023 & 0.471 & $0.112-1.975$ & 0.3035 \\
\hline Grade & 0.868 & $0.187-4.030$ & 0.8564 & 1.363 & $0.647-2.874$ & 0.4155 \\
\hline HR_Status ER(+ve)/Her2(-ve) & 8.981 & $0.774-104.259$ & 0.0793 & 0.649 & $0.283-1.489$ & 0.3079 \\
\hline HR_Status ER(-ve)/Her2(+ve) & 31.436 & $2.109-468.544$ & 0.0124 & 0.412 & $0.102-1.662$ & 0.2096 \\
\hline HR_Status ER(-ve)/Her2(-ve) & 30.258 & $2.054-445.803$ & 0.0130 & 0.592 & $0.161-2.231$ & 0.4445 \\
\hline ER_PR_Status ER(+ve)/PR(-ve) & 0.074 & $0.003 .009-1.771$ & 0.1080 & 0.759 & $0.110-5.254$ & 0.7802 \\
\hline ER_PR_Status ER(+ve)/PR(+ve) & 0.845 & $0.122-5.837$ & 0.8640 & 0.726 & $0.169-3.108$ & 0.6655 \\
\hline \multirow[t]{2}{*}{ Parameter } & \multicolumn{3}{|l|}{ Overall survival } & \multicolumn{3}{|c|}{ Disease-free survival } \\
\hline & Hazard ratio & $95 \% \mathrm{Cl}+$ & $P^{*}$ & Hazard ratio & $95 \% \mathrm{Cl}+$ & $P^{*}$ \\
\hline ATR (epithelial cells) & 10.242 & $1.745-60.111$ & 0.0100 & 0.834 & $0.436-1.598$ & 0.5849 \\
\hline Tumor size & 0.049 & $0.006-0.425$ & 0.0062 & 1.459 & $0.402-5.301$ & 0.5659 \\
\hline Age & 0.880 & $0.804-0.962$ & 0.0050 & 1.028 & $0.989-1.068$ & 0.1637 \\
\hline Stage & 257.360 & $13.853-4781.270$ & 0.0002 & 0.334 & $0.084-1.325$ & 0.1188 \\
\hline Grade & 1.205 & $0.266-5.452$ & 0.8083 & 1.379 & $0.672-2.832$ & 0.3807 \\
\hline HR_Status ER(+ve)/Her2(-ve) & 2.731 & $0.382-19.517$ & 0.3166 & 0.633 & $0.266-1.505$ & 0.3004 \\
\hline HR_Status ER(-ve)/Her2(+ve) & 1.456 & $0.146-14.486$ & 0.7488 & 0.657 & $0.196-2.207$ & 0.4973 \\
\hline HR_Status ER(-ve)/Her2(-ve) & 4.817 & $0.459-50.592$ & 0.1901 & 0.655 & $0.193-2.225$ & 0.4973 \\
\hline ER_PR_Status ER(+ve)/PR(-ve) & 0.901 & $0.465-25.058$ & 0.9296 & 1.187 & $0.207-6.820$ & 0.8474 \\
\hline ER_PR_Status ER(+ve)/PR(+ve) & 10.511 & $0.671-164.698$ & 0.2275 & 1.021 & $0.983-1.061$ & 0.2730 \\
\hline
\end{tabular}

clear indication that low levels of ATR in tumor cells as well as their adjacent stromal fibroblasts predict high risk of recurrence and poor survival postneoadjuvant treatment of locally advanced breast cancer patients. Therefore, the level of this protein kinase in breast cancer cells or in the adjacent stromal fibroblasts could constitute a powerful prognostic biomarker for these hard-to-treat patients who need downstaging tumors to facilitate breast conservation therapy.

\section{Supplementary information}

Supplementary information accompanies this paper at https://doi.org/10. 1186/s13058-020-01289-4.

Additional file 1. Clinicopathological characteristics of the breast cancer patients. This table summarizes the clinicopathological features of the patients.

Additional file 2. Expression of ATR in cancer cells and stromal fibroblasts. This table is showing the expression of ATR in four different breast cancer sub-types.

\section{Abbreviations}

ATR: Ataxia telangiectasia and Rad3-related; BSF: Breast stromal fibroblast; CAF: Cancer-associated fibroblast; DFS: Disease-free survival; IL-6: Interleukin6; LABC: Locally advanced breast cancer; OS: Overall survival; SFM: Serum-free media; SFCM: Serum-free conditioned media; TCF: Tumor counterpart fibroblast

\section{Acknowledgements}

We are grateful to Dr. Myriam Gorospe for kindly providing us with the pSILENCER-AUF15 plasmid. We are also thankful to Mrs. Fauziah Hendrayani for her technical support. This work was performed under the RAC proposal \# 2120034.

Authors' contributions

AA and KK contributed to the conception and design; MA-A, MA-S, AE, AS, $A T$, and MA contributed to the development of methodology and acquisition of data; MA-A, TA-T, and AA contributed to the analysis and interpretation of data; MA-A, KK, AS, and AA contributed to the writing of the manuscript. The author(s) read and approved the final manuscript.

Funding

This work was solely supported by the King Faisal Specialist Hospital and Research Centre.

Availability of data and materials

The data generated, used, and analyzed in the current study are available from the corresponding author in response to a reasonable request.

Ethics approval and consent to participate

Written informed consent was not required and a waiver was granted since the study was retrospective and samples were anonymized to the research team. This was granted by the institutional review board approval (RAC\#2160005). 


\section{Consent for publication}

Not applicable.

\section{Competing interests}

The authors declare that they have no competing interests.

\section{Author details}

'Department of Molecular Oncology, King Faisal Specialist Hospital and Research Center, MBC\#03, Riyadh 11211, Saudi Arabia. ${ }^{2}$ Department of Microbiology, Faculty of Science and Medical Studies, King Saud University, Riyadh, Saudi Arabia. ${ }^{3}$ Molecular BioMedicine Program, Research Centre, King Faisal Specialist Hospital and Research Centre, Riyadh 11211, Saudi Arabia. ${ }^{4}$ Department of Pathology, King Saud University, PO BOX 2925, Riyadh 11461, Saudi Arabia. ${ }^{5}$ Department of Biostatistics, Epidemiology and Scientific computing, King Faisal Specialist Hospital and Research Center, Riyadh 11211, Saudi Arabia. ${ }^{6}$ Department of Pathology, King Faisal Specialist Hospital and Research Center, Riyadh 11211, Saudi Arabia. 'Department of Oncology, King Faisal Specialist Hospital and Research Center, Riyadh 11211, Saudi Arabia. ${ }^{8}$ Groupe de Recherche en Écologie Buccale, Faculté de Médecine Dentaire, Université Laval Québec, Local 1758, 2420 rue de la terrasse, Québec G1V 0A6, Canada.

Received: 30 November 2019 Accepted: 6 May 2020

Published online: 15 May 2020

\section{References}

1. Guarneri V, Broglio K, Kau SW, Cristofanilli M, Buzdar AU, Valero V, et al. Prognostic value of pathologic complete response after primary chemotherapy in relation to hormone receptor status and other factors. J Clin Oncol. 2006;24:1037-44.

2. Aboussekhra A. Role of cancer-associated fibroblasts in breast cancer development and prognosis. Int J Dev Biol. 2011;55:841-9.

3. Kalluri R. The biology and function of fibroblasts in cancer. Nat Rev Cancer 2016;16:582-98.

4. Chen X, Song E. Turning foes to friends: targeting cancer-associated fibroblasts. Nat Rev Drug Discov. 2018;18:99-115.

5. Andor N, Maley CC, Ji HP. Genomic instability in cancer: teetering on the limit of tolerance. Cancer Res. 2017;77:2179-85.

6. Lecona E, Fernandez-Capetillo O. Targeting ATR in cancer. Nat Rev Cancer. 2018;18:586-95

7. Weber AM, Ryan AJ. ATM and ATR as therapeutic targets in cancer. Pharmacol Ther. 2015;149:124-38.

8. Al-Ansari MM, Aboussekhra A. ATR suppresses the pro-tumorigenic functions of breast stromal fibroblasts. Oncotarget. 2018;9:34681-90.

9. Hawsawi NM, Ghebeh H, Hendrayani SF, Tulbah A, Al-Eid M, Al-Tweigeri T, et al. Breast carcinoma-associated fibroblasts and their counterparts display neoplastic-specific changes. Cancer Res. 2008;68:2717-25.

10. Al-Mohanna MA, Al-Khalaf HH, Al-Yousef N, Aboussekhra A. The p16INK4a tumor suppressor controls p21WAF1 induction in response to ultraviolet light. Nucleic Acids Res. 2007;35:223-33.

11. Wang W, Martindale JL, Yang X, Chrest FJ, Gorospe M. Increased stability of the p16 mRNA with replicative senescence. EMBO Rep. 2005;6:158-64.

12. Al-Saif M, Khabar KS. UU/UA dinucleotide frequency reduction in coding regions results in increased mRNA stability and protein expression. Mol Ther. 2012;20:954-9.

13. Patel N, Khan AO, Al-Saif M, Moghrabi WN, AlMaarik BM, Ibrahim N, et al. A novel mechanism for variable phenotypic expressivity in Mendelian diseases uncovered by an AU-rich element (ARE)-creating mutation. Genome Biol. 2017;18:144.

14. Semlali A, Chakir J, Goulet JP, Chmielewski W, Rouabhia M. Whole cigarette smoke promotes human gingival epithelial cell apoptosis and inhibits cell repair processes. J Periodontal Res. 2011;46:533-41.

15. Semlali A, Chakir J, Rouabhia M. Effects of whole cigarette smoke on human gingival fibroblast adhesion, growth, and migration. J Toxicol Environ Health A. 2011;74:848-62.

16. Semlali A, Reddy Parine N, Arafah M, Mansour L, Azzi A, Al Shahrani O, et al. Expression and polymorphism of toll-like receptor 4 and effect on NFkappaB mediated inflammation in colon cancer patients. PLoS One. 2016;11: e0146333.

17. Hendrayani SF, Al-Khalaf HH, Aboussekhra A. The cytokine IL-6 reactivates breast stromal fibroblasts through transcription factor STAT3- dependent up-regulation of the RNA-binding protein AUF1. J Biol Chem. 2014;289:30962-76.

18. Gieniec KA, Butler LM, Worthley DL, Woods SL. Cancer-associated fibroblasts-heroes or villains? Br J Cancer. 2019;121:293-302.

19. Togo S, Polanska UM, Horimoto Y, Orimo A. Carcinoma-associated fibroblasts are a promising therapeutic target. Cancers (Basel). 2013;5: 149-69.

20. Al-Ansari MM, Aboussekhra A. miR-146b-5p mediates p16-dependent repression of IL-6 and suppresses paracrine procarcinogenic effects of breast stromal fibroblasts. Oncotarget. 2015;6:30006-16.

21. Al-Ansari MM, Hendrayani SF, Shehata Al, Aboussekhra A. p16(INK4A) represses the paracrine tumor-promoting effects of breast stromal fibroblasts. Oncogene. 2013;32:2356-64.

22. Lal A, Mazan-Mamczarz K, Kawai T, Yang X, Martindale JL, Gorospe M. Concurrent versus individual binding of HuR and AUF1 to common labile target mRNAs. EMBO J. 2004;23:3092-102.

\section{Publisher's Note}

Springer Nature remains neutral with regard to jurisdictional claims in published maps and institutional affiliations.
Ready to submit your research? Choose BMC and benefit from:

- fast, convenient online submission

- thorough peer review by experienced researchers in your field

- rapid publication on acceptance

- support for research data, including large and complex data types

- gold Open Access which fosters wider collaboration and increased citations

- maximum visibility for your research: over $100 \mathrm{M}$ website views per year

At $\mathrm{BMC}$, research is always in progress.

Learn more biomedcentral.com/submissions 